\title{
Effectiveness and Safety of Xinkeshu Tablet (XKS) on Coronary Heart Disease Patients Combined with Anxiety and Depression Symptoms After Percutaneous Coronary Intervention: A Protocol for Systematic Review and Meta-Analysis
}

\section{Mingtai Chen ( $\nabla$ zyycardio@foxmail.com )}

Shenzhen traditional Chinese medicine hospital https://orcid.org/0000-0003-4579-5559

\section{Yingnan Chen}

Shenzhen traditional Chinese medicine hospital

\section{Ling Men}

Shenzhen traditional Chinese medicine hospital

\section{Xiaoling Zhong}

Shenzhen traditional Chinese medicine hospital

\section{Shudong Yang}

Shenzhen traditional Chinese medicine hospital

Jienan Luan

Shenzhen traditional Chinese medicine hospital

\section{Protocol}

Keywords: Xinkeshu, percutaneous coronary intervention, anxiety and depression, randomized trial, metaanalysis, protocol

Posted Date: July 20th, 2020

DOl: https://doi.org/10.21203/rs.3.rs-44377/v1

License: (c) (i) This work is licensed under a Creative Commons Attribution 4.0 International License.

Read Full License 


\section{Abstract}

Backgroud: It's known that coronary heart disease (CHD) patients after percutaneous coronary intervention (PCl) was significantly associated with anxiety and depression symptoms. Several studies have showed that Xinkeshu tablet (XKS), a kind of Chinese herbal medicine (CHM), could effectively improve post- $\mathrm{PCl}$ postoperative mood disorders in CHD patients. However, the intensity of evidence has been poor, limiting the further clinical application of XKS to patients above. This systematic review and meta-analysis will assess studies of the effectiveness and safety of XKS in CHD patients with anxiety and depression symptoms after $\mathrm{PCl}$.

Methods: A systematic literature search for articles up to July 2020 will be performed in following electronic databases: PubMed, Embase, the Cochrane Library, China National Knowledge Infrastructure (CNKI), Chinese Scientific Journals Database (VIP) Database, Chinese Biomedical Database (CBM), Chinese Biomedical Literature Service System (SinoMed) and Wanfang Database. Inclusion criteria are randomized controlled trials of XKS applied on patients with CHD and depression. The primary outcome measures will be CHD-related clinical evaluation (frequency of acute attack angina, severity of angina pectoris, electrocardiographic changes, amount of nitroglycerin) and the scores or reducing fractions of depressive and anxiety measuring scales (the Hospital Anxiety / Depression Scale or other widely used anxiety / depression scale). The safety outcome measures will be adverse events, liver and kidney function. RevMan 5.3 software will be used for data synthesis, sensitivity analysis, subgroup analysis and risk of bias assessment. A funnel plot will be developed to evaluate reporting bias. Stata 12.0 will be used for meta-regression and Egger tests. We will use the Grading of Recommendations Assessment, Development and Evaluation (GRADE) system to assess the quality of evidence.

Discussion: This study will provide a high-quality synthesis of the effects and safety of XKS for CHD patients with anxiety and depression symptoms after $\mathrm{PCI}$.

Ethics and dissemination This systematic review does not require ethics approval and will be submitted to a peer-reviewed journal.

\section{Trial registration number PROSPERO CRD42019131346.}

\section{Backgroud}

Anxiety and depression symptoms are predictors of a worse prognosis in coronary heart disease (CHD) patients $^{1-4}$. It was confirmed that $\mathrm{CHD}$ patients after percutaneous coronary intervention(PCI) was significantly associated with anxiety and depression symptoms at different follow-up time points ${ }^{5}$. In addition, anxiety and depression symptoms of CHD patients aggravated significantly after $\mathrm{PCl}^{6}$. In the population of $\mathrm{CHD}$ patients after $\mathrm{PCl}$, anxiety and depression symptoms not only were associated with a increased risk for major adverse cardiac events but were shown to severely disrupt long-term quality of life ${ }^{47}$. As far as the severity of this issuse was concerned, several therapeutic measures were tried to 
solve this problem, such as anti-anxiety / anti-depression therapy, Tai Chi, cardiac rehabilitation (CR),

etc $^{8-10}$. However, the results were dissatisfying, in which the side effects of anti-anxiety / antidepressants therapy limited their use overall in the long-term treatment of CHD patients and CR couldn't significantly improve anxiety/depression of CHD patients ${ }^{11-12}$.

Chinese herbal medicine (CHM) has been used clinically as therapy treatment for thousands of years. Xinkeshu tablet (XKS) as a complex matrix comprises five medicinal materials or extracts thereof, including Salvia miltiorrhiza Bge, Radix Puerariae, Hawthorn, Panax Notoginseng and Radix Aucklandiae. XKS, which was proven anti-anxiety effects and associated with little toxicity, was widely applied as an alternative therapy for the CHD combined with anxiety and depression in China. Several studies showed that XKS could effectively improve post-PCI postoperative mood disorders in patients with $\mathrm{CHD}$ and improve the quality of life $\mathrm{e}^{15-17}$.

Although numerous clinical studies and reviews have assessed XKS in the treatment of CHD patients with anxiety and depression, systematic review to evaluate the effectiveness and safety of XKS in such patient population was rare. We identified only one similar meta-analysis evaluating the effectiveness of XKS therapies in CHD patients with anxiety and depression, though the study had certain limitations ${ }^{18}$. There were 18 randomized clinical trials (RCTs) included in the meta-analysis by Yuan et al. However, the primary outcome measures of the CHD-related clinical evaluations and anxiety/depression score scale were relatively insufficient; and the subgroup analysis about whether the patients experienced PCI treatment were not conducted. In view of the shortcomings of previous studies and the incomplete evidence regarding the widespread use of XKS, this meta-analysis aimed to summarize the effectiveness and safety of XKS in treating CHD patients with anxiety and depression after $\mathrm{PCl}$.

\section{Methods And Analysis}

\section{Registration}

The study protocol has been registered in the international prospective register of systematic review (PROSPERO). The trial registration number of PROSPERO is CRD42019131346. The procedure of this protocol will be conducted according to the Preferred Reporting Item for Systematic Review and Metaanalysis Protocols (PRISMA-P) guidelines. ${ }^{19}$

\section{Eligibility criteria}

\section{Type of study}

Inclusion: We will include all the RCTs that investigated the effectiveness and safety of XKS combined with conventional pharmacotherapy for the treatment of $\mathrm{CHD}$ patients with anxiety and depression after $\mathrm{PCl}$. 
Exclusion: The studies will be excluded if it is not an RCT (namely, observational cohort and case-control studies, case reports, experimental studies and reviews ).

\section{Participants}

Inclusion: The study will include adult (18-85years) CHD patients with anxiety and depression after PCI regardless of sex, ethnicity, education or economic status and whether or not they were out- or in-patients. The diagnostic criteria for $\mathrm{CHD}$, anxiety and depression will be as follows.

1. The diagnostic criteria of CHD should be confirmed according to one of the past or current definitions: Report of the Joint International Society and Federation of Cardiology (IFSC) / World Health Organization (WHO) task force on standardization of clinical nomenclature of ischaemic heart disease, or the American College of Cardiology (ACC) / American Heart Association (AHA) guideline update for the management of patients with chronic stable angina or Chinese Association

of Cardiology or unstable angina pectoris diagnosis and treatment recommendations . $^{20-22}$

2. Anxiety and depression must be defined as anxiety disorder or clinical anxiety and depressive disorder or clinical depression diagnosed according to the Diagnostic and Statistical Manual of Mental Disorders (DSM), the International Classification of Diseases (ICD) by a standardized interview (e.g., Structured Clinical Interview, Composite International Diagnostic Interview) or the Chinese Classification of Mental Disorders (CCMD). ${ }^{23-25}$

Exclusion: Patients with either CHD or depression or anxiety only will be excluded. Patients with severe respiratory disease, acute infectious disease, severe heart disease, severe liver disease or tumors will be excluded.

\section{Interventions}

Inclusion: Eligible interventions will be those involving a combination of XKS and conventional pharmacotherapy. The same conventional pharmacotherapy must be used in the control group.

Exclusion: Trials that include other co-interventions such as another herbal formula, acupuncture, cupping, moxibustion, massage, yoga, qigong, Tai Chi, or aromatherapy will be excluded.

\section{Outcome}

Inclusion: The primary outcome measures will include the following: CHD-related clinical evaluation (frequency of acute angina, severity of angina pectoris, electrocardiographic changes, dose of nitroglycerin), the scores or reduction in scales measuring depression and anxiety (i.e., the Hospital Anxiety and Depression Scale or other widely used anxiety / depression scale). The secondary outcome measures will include the following: total cholesterol(TC), triglyceride (TG), low-density lipoprotein 
cholesterol (LDL-C) and high-density lipoprotein cholesterol (HDL-C) levels, and the Traditional Chinese Medicine (TCM) syndrome scale. The safety outcomes will include the following: adverse events (such as digestive symptoms, headache, dizziness, skin rash etc.), liver or kidney toxicity measured by serum markers.

Exclusion: The outcome measures not requested in this study will be excluded.

\section{Search strategy}

The following electronic bibliographic databases will be searched from inception to July 2020: PubMed, Embase, the Cochrane Library, China National Knowledge Infrastructure (CNKI), Chinese Scientific Journals Database (VIP) Database, Chinese Biomedical Database (CBM), Chinese Biomedical Literature Service System (SinoMed) and Wanfang Database. A manual search of key journals and of the reference lists of reviews captured by the initial searches will also be performed. There will be no limits on the language of publication. Only clinical trials will be included and searched. The following sources will also be searched to identify clinical trials that are in progress or completed: Clinical Trials.gov and WHO clinical trials registry. Any additional relevant studies will also be retrieved from the reference lists of systematic reviews and included studies. If possible, we will map search terms to controlled vocabulary. In addition, the search strategy for selecting the fields of title, abstract or keyword will differ depending on the characteristics of the databases. Search terms will be grouped into three blocks (see Table 1). 
Table 1

Search items

\section{Search Search items \\ Block}

Participants (Coronary Disease OR Myocardial Ischemia OR Coronary Diseases OR Disease, Coronary OR Diseases, Coronary OR Coronary Heart Disease OR Coronary Heart Diseases OR Disease, Coronary Heart OR Diseases, Coronary Heart OR Heart Disease, Coronary OR Heart Diseases, Coronary OR CHD OR Myocardial Infarction OR Cardiovascular Stroke OR Heart Attack OR Myocardial Infarct OR Acute Coronary Syndrome OR Angina Pectoris OR Angina, Stable OR Angina, Unstable OR Angina Pectoris, Variant OR Microvascular Angina)

\section{AND}

(Depressions OR Depressive Symptoms OR Depressive Symptom OR Symptom, Depressive OR Symptoms, Depressive OR Emotional Depression OR Depression, Emotional OR Depressions, Emotional OR Emotional Depressions OR Depressive Disorders, Major OR Depressive Disorders OR Depressive Syndrome )

AND

(Anxiety OR Anxiety Symptoms OR Anxiety Symptom OR Symptom, Anxiety OR Symptoms, Anxiety OR Nervousness OR Social Anxiety OR Anxieties, Social OR Anxiety, Social OR Social Anxieties OR Anxiety Disorder OR Disorder, Anxiety OR Disorders, Anxiety OR Neuroses, Anxiety OR Anxiety Neuroses OR Anxiety States, Neurotic OR Anxiety State, Neurotic OR Neurotic Anxiety State OR Neurotic Anxiety States OR State, Neurotic Anxiety OR States, Neurotic Anxiety)

Intervention Xinkeshu OR Xin Ke Shu OR XKS OR Xinkeshu tablet OR Xinkeshu capsule OR XKS tablet OR XKS capsule OR Xin Ke Shu tablet OR Xin Ke Shu capsule OR Drugs, Chinese Herbal OR Chinese Drugs, Plant OR Chinese Herbal Drugs OR Herbal Drugs, Chinese OR Plant Extracts, Chinese OR Chinese Plant Extracts OR Extracts, Chinese Plant OR Medicine, Chinese Traditional OR Traditional Chinese Medicine OR Chung I Hsueh OR Hsueh, Chung I OR Traditional Medicine, Chinese OR Zhong Yi Xue OR Chinese Traditional Medicine OR Chinese Medicine, Traditional

Study design
Randomized controlled trial OR controlled clinical trial OR randomized OR placebo OR drug therapy OR randomly OR trial OR groups

\section{Study selection and data extraction}

Literature retrieved citations will be managed by EndNote X7 software. Two authors (MC and YNC) will independently screen the titles and abstracts of all the studies retrieved in the above electronic databases to identify potentially eligible studies. Articles that are duplicated or have not met the eligibility criteria, interventions and outcomes in this study will be excluded. After filtering the final eligible articles, the data from the included articles will be extracted independently by two authors (MC and LM). Disagreements will be resolved by discussion or arbitrated by a third author if needed. The following categories of data will be extracted: first author, publication year, diagnose information, age, sex, trial characteristics, interventions and controls, participants, study methodology, outcomes, and adverse events (see Fig. 1).

\section{Risk of bias assessment}


The methodological quality of the eligible studies will be evaluated according to the Cochrane Collaboration's tool for assessing risk of bias. The assessment details include: sequence generation, allocation concealment, blinding of participants and personnel, blinding of outcome assessors, incomplete outcome data, selective reporting and other sources of bias. Each domain will be assessed as "low risk", "high risk" or "unclear risk" according to the description details of eligible studies.

\section{Data synthesis and statistical analysis}

Statistical analyses will be conducted with RevMan 5.3 software provided by Cochrane Collaboration. The overall effect sizes will be determined as the mean difference (MD) for continuous outcomes, the odds ratio (OR) for dichotomous outcomes with their $95 \%$ credible intervals (Cls). The $\mathrm{Q}$ and $\mathrm{I}^{2}$ test statistics will be calculated to determine the amount of heterogeneity. For the Q statistic, $p<0.05$ will be considered to indicate significant differences. For the $\mathrm{I}^{2}$ statistic, $\mathrm{I}^{2}<25 \%$ indicates no significant heterogeneity, $\mathrm{I}^{2}=25-50 \%$ is considered moderate heterogeneity and $\mathrm{I}^{2}>50 \%$ indicates strong heterogeneity. We will use fixed effects models if there is no heterogeneity among studies, and random effects models if there is heterogeneity.

\section{Sensitivity analysis, subgroup analysis and meta-regression}

If the heterogeneity or inconsistency among the studies is detected, a sensitivity analysis or subgroup analysis or meta-regression (conducted by Stata 12.0) analysis will be performed. Subgroup analysis will be conducted to explore potential sources of heterogeneity according to the characteristics of studies, including sample size, types of CHD, severity of depression, severity of anxiety, dose of XKS, treatment duration and other relevant parameters. If data extraction is insufficient, we will create a qualitative synthesis.

\section{Publication bias}

A funnel plot will be developed to evaluate reporting bias of the included studies. We will use Egger tests (conducted by Stata 12.0) to assess funnel plot symmetry and will interpret values of $p<0.1$ as statistically significant.

\section{Quality of evidence}

We will also assess the quality of evidence for the main outcomes with the Grading of

Recommendations Assessment, Development and Evaluation (GRADE) approach. Five items will be investigated, including limitations in study design, inconsistency, inaccuracies, indirectness and publication bias.

\section{Patient and Public Involvement}

The patients and / or public will not be involved because this study uses secondary sources for analysis.

\section{Disscussion}


We plan to conduct this meta-analysis to assess the effectiveness and safety of XKS for CHD patients with anxiety and depression after PCl. However, there may be some limitations because this is a retrospective meta-analysis. First, during the search, there is the inevitable potential that unpublished studies will not be identified which will introduce some bias. Besides, some grey literature may be difficult to retrieve, possibly leading to a selection bias in the literature. Moreover, some secondary outcome measures may not be completely reported. However, we expect that the results of this study will be able to propose clinical recommendations for $\mathrm{CHD}$ patients with anxiety and depression after $\mathrm{PCl}$ in clinical practices and provide more objective and reliable evidence supporting use of the XKS.

\section{Abbreviations}

CHD: coronary heart disease; PCl: percutaneous coronary intervention; XKS: Xinkeshu tablet; CHM: Chinese herbal medicine; CNKI: China National Knowledge Infrastructure; VIP: Chinese Scientific Journals Database Database; CBM: Chinese Biomedical Database; SinoMed: Chinese Biomedical Literature Service System; GRADE: Grading of Recommendations Assessment, Development and Evaluation;

PRISMA-P: Preferred Reporting Item for Systematic Review and Meta-analysis Protocols; IFSC: Joint International Society and Federation of Cardiology (IFSC) ; WHO: World Health Organization; ACC: the American College of Cardiology; AHA: American Heart Association (AHA); DSM: Diagnostic and Statistical Manual of Mental Disorders; ICD: the International Classification of Diseases; CCMD: Chinese Classification of Mental Disorders; TC: total cholesterol; TG: triglyceride ; LDL-C: low-density lipoprotein cholesterol; HDL-C: high-density lipoprotein cholesterol; MD: mean difference; OR: odds ratio; Cl: credible interval.

\section{Declarations}

\section{Ethical Approval and Consent to participate}

No ethics approval is required for this systematic review and meta-analysis because we will be using information from published studies. Our findings will be published in a peer-reviewed journal according to the PRISMA guidelines.

\section{Consent for publication}

Not applicable.

\section{Availability of supporting data}

Not applicable. 


\section{Competing interests}

None declared.

\section{Funding}

This study is supported by National Natural Science Foundation of China, NFSC \No.81573922ð; Sanming Project of Medicine in Shenzhen - Chinese Academy of Medical Sciences Fuwai Hospital Professor Zhang Jian Cardiovascular Disease Team 囚No.SZSM201612033囚

\section{Authors' contributions}

$\mathrm{JL}$ and SY conceived the study and drafted the protocol. $\mathrm{JL}$ and $\mathrm{XZ}$ revised it. MC, YC and LM developed the search strategies, will conduct data collection and analyse the data independently. All authors will approve the final

\section{Acknowledgements}

Not applicable.

\section{Authors' information}

Mingtai Chen1, Yingnan Chen1, Ling Men2, Xiaoling Zhong3, Shudong Yang2, Jienan Luan1

1. Department of Cardiovascular Disease, Shenzhen Traditional Chinese Medicine Hospital, Shenzhen, China

2. Nephrology Department, Shenzhen Traditional Chinese Medicine Hospital, Shenzhen, China;

3. Reproductive Health Department, Shenzhen Traditional Chinese Medicine Hospital, Shenzhen, China

Correspondence to

Dr. Mingtai Chen: zyycardio@foxmail.com

Professor Shudong Yang: szszyyysd@163.com

Professor Jienan Luan: Iuanjienan@sohu.com

\section{References}

1. Xiao Y, Li W, Zhou J, et al. Impact of depression and/or anxiety on patients with percutaneous coronary interventions after acute coronary syndrome: a protocol for a real-world prospective cohort 
study. BMJ OPEN. 2019;9(9):e27964. doi:10.1136/bmjopen-2018-027964.

2. Brand A, Gao L, Hamann A, et al. Medical Graphic Narratives to Improve Patient Comprehension and Periprocedural Anxiety Before Coronary Angiography and Percutaneous Coronary Intervention: A Randomized Trial. ANN INTERN MED. 2019;170(8):579-81. doi:10.7326/M18-2976.

3. Celano CM, Millstein RA, Bedoya CA, et al. Association between anxiety and mortality in patients with coronary artery disease: A meta-analysis. AM HEART J. 2015;170(6):1105-15. doi:10.1016/j.ahj.2015.09.013.

4. $10.1161 /$ CIR. 0000000000000019 Lichtman JH, Froelicher ES, Blumenthal JA, et al. Depression as a risk factor for poor prognosis among patients with acute coronary syndrome: systematic review and recommendations: a scientific statement from the American Heart Association. CIRCULATION 2014;129(12):1350-69. doi: 10.1161/CIR.0000000000000019.

5. Aragam KG, Moscucci M, Smith DE, et al. Trends and disparities in referral to cardiac rehabilitation after percutaneous coronary intervention. AM HEART J. 2011;161(3):544-51. doi: 10.1016/j.ahj.2010.11.016.

6. Beatty AL, Doll JA, Schopfer DW, et al. Cardiac Rehabilitation Participation and Mortality After Percutaneous Coronary Intervention: Insights From the Veterans Affairs Clinical Assessment, Reporting, and Tracking Program. J AM HEART ASSOC. 2018;7(19):e10010. doi:10.1161/JAHA.118.010010.

7. Samartzis L, Dimopoulos S, Tziongourou M, Nanas S. Effect of psychosocial interventions on quality of life in patients with chronic heart failure: a meta-analysis of randomized controlled trials. J CARD FAIL. 2013;19(2):125-34. doi:10.1016/j.cardfail.2012.12.004.

8. Liu J, Yu P, Lv W, Wang X. The 24-Form Tai Chi Improves Anxiety and Depression and Upregulates miR-17-92 in Coronary Heart Disease Patients After Percutaneous Coronary Intervention. FRONT PHYSIOL. 2020;11:149. doi:10.3389/fphys.2020.00149.

9. Kotseva K, Wood D, De Bacquer D. Determinants of participation and risk factor control according to attendance in cardiac rehabilitation programmes in coronary patients in Europe: EUROASPIRE IV survey. EUR J PREV CARDIOL. 2018;25(12):1242-51. doi:10.1177/2047487318781359.

10. Olsen SJ, Schirmer H, Wilsgaard T, Bønaa KH, Hanssen TA. Cardiac rehabilitation and symptoms of anxiety and depression after percutaneous coronary intervention. EUR J PREV CARDIOL. 2018;25(10):1017-25. doi:10.1177/2047487318778088.

11. Wang AL, Chen Z, Luo J, Shang QH, Xu H. Systematic review on randomized controlled trials of coronary heart disease complicated with depression treated with Chinese herbal medicines. CHIN J INTEGR MED. 2016;22(1):56-66. doi:10.1007/s11655-015-2110-9.

12. Nedeljkovic I. Assessment of depression and anxiety in patients before and after percutaneous coronary intervention: A step forward in cardiac rehabilitation? EUR J PREV CARDIOL. 2018;25(10):1015-16. doi:10.1177/2047487318774094. 
13. He Dequan Li Wenjie Tan Wenpeng. Clinical observation of Xinkeshu in the treatment of anxiety in patients with acute coronary syndromeafter PCl. Cardiovascular Disease Electronic Journal of integrated traditional Chinese Western Medicine. 2019;7(23):5-06.

14. Zhang Jiehan Zhuang Shaowei Tong Shihua. Clinical Efficacy of Xinkeshu Tablets in the treatment of Coronary Heart Disease Patients with Depression after PCl and Effect on the Quality of Life. Progress in Modern Biomedicine. 2018;18(2):305-25.

15. Yang Guanglong Yin Qiong Wang Shuping. Effects of Xinkeshu tablet on patients with coronary heart disease, anxiety and depression after PCl. Chinese Journal of Integrative Medicine on Cardio-/Cerebrovascuiar Disease. 2013;11(11):1323.

16. Dai Xiaohua Yuan Liang. Efficacy of Xinkeshu Tablet in the treatment of coronary heart disease with anxiety depression: a meta-analysis. Chinese Journal of Integrative Medicine on Cardio-/Cerebrovascuiar Disease. 2017;15(7):772-76.

17. Moher $D$, Shamseer $L$, Clarke $M$, et al. Preferred reporting items for systematic review and metaanalysis protocols (PRISMA-P) 2015 statement. Systematic reviews. 2015;4:1. doi:10.1186/20464053-4-1.

\section{Tables}

\section{Strengths and limitations of this study}

This study is a objective and normative systematic review on the effectiveness and safety of modified Xinkeshu tablet as a kind of Chinese herbal medicine for treating CHD patients with anxiety and depression after PCl.

Study screening, data extraction and assessment of the risk of bias will be independently conducted by two researchers

Disagreements will be resolved by discussion or arbitrated by a third author if needed

There may be clinical heterogeneity because of variations in treatment frequency or duration or other factors. Therefore, subgroup analysis or metaregression analysis will be carried out to explore any sources of heterogeneity.

The Grading of Recommendations Assessment,

Development and Evaluation system will be used to further evaluate study findings.

Because this is a retrospective meta-analysis, the search will be performed in our study and potential unpublished trials will be inevitable which will introduce some bias.

\section{Figures}




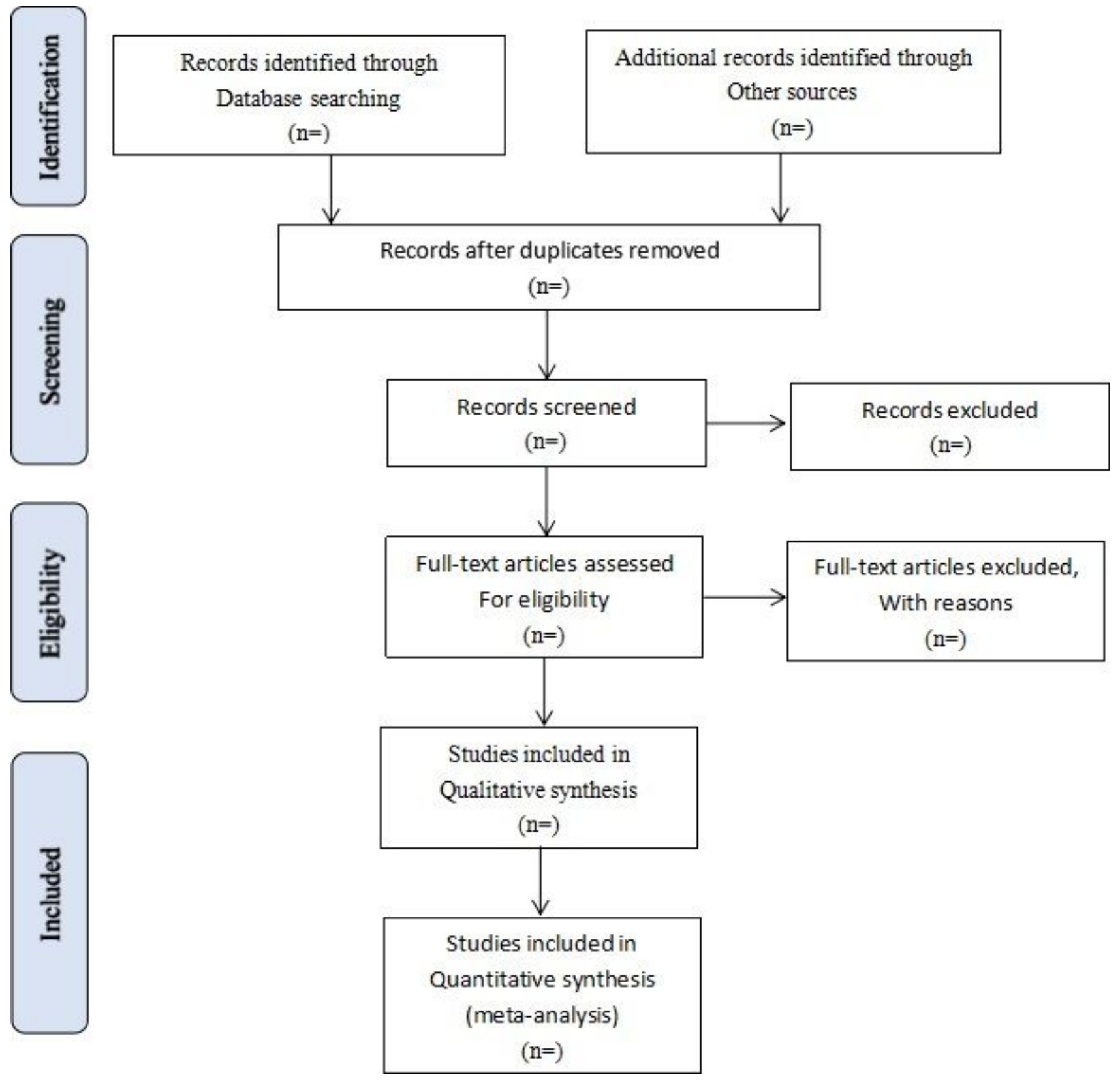

Figure 1

Flow diagram of study selection process. PubMed, Embase, the Cochrane Library, CNKI, VIP Database, CBM, SinoMed and Wanfang Database.

\section{Supplementary Files}

This is a list of supplementary files associated with this preprint. Click to download.

- Sammingproject.pdf

- renamed03300.pdf

- PRISMAP.doc 